\title{
Nonlinear Eigenvalue Problem for Optimal Resonances in Optical Cavities
}

\author{
I.M. Karabash * \\ Institute of Applied Mathematics and Mechanics of NAS of Ukraine \\ R. Luxemburg str. 74, Donetsk 83114, Ukraine
}

\begin{abstract}
The paper is devoted to optimization of resonances in a 1-D open optical cavity. The cavity's structure is represented by its dielectric permittivity function $\varepsilon(s)$. It is assumed that $\varepsilon(s)$ takes values in the range $1 \leq \varepsilon_{1} \leq \varepsilon(s) \leq \varepsilon_{2}$. The problem is to design, for a given (real) frequency $\alpha$, a cavity having a resonance with the minimal possible decay rate. Restricting ourselves to resonances of a given frequency $\alpha$, we define cavities and resonant modes with locally extremal decay rate, and then study their properties. We show that such locally extremal cavities are 1-D photonic crystals consisting of alternating layers of two materials with extreme allowed dielectric permittivities $\varepsilon_{1}$ and $\varepsilon_{2}$. To find thicknesses of these layers, a nonlinear eigenvalue problem for locally extremal resonant modes is derived. It occurs that coordinates of interface planes between the layers can be expressed via arg-function of corresponding modes. As a result, the question of minimization of the decay rate is reduced to a four-dimensional problem of finding the zeroes of a function of two variables.
\end{abstract}

Keywords and phrases: photonic crystal, high Q-factor resonator, quasi-normal eigenvalue optimization, nonlinear eigenvalue

Mathematics Subject Classification: 78M50, 49R05, 47N50, 47A55

\section{Introduction}

A leaky optical cavity (or resonator) is a region of space within which the electromagnetic field is well confined, but not completely confined. Because of the leakage, each standing electromagnetic wave in the cavity decays exponentially in time. The rate of energy loss and the frequency of these eigenoscillations can be characterized by the corresponding complex eigenvalue $\omega$, which is called a quasi-normal (QN) eigenvalue or a resonance (the latter name is used in Quantum Mechanics and, sometimes, Engineering). The imaginary part $\operatorname{Im} \omega$ of the QN eigenvalue corresponds to the decay rate of the standing wave, the real part Re $\omega$ to the frequency of oscillations.

Optical cavities with small and high decay rate are required in Optical Engineering because they enhance intrinsically small light-matter interactions. For example, optical microcavities with very small decay rates are used in strong-coupling cavity quantum electrodynamics to ascertain atomic center-

\footnotetext{
${ }^{*}$ Corresponding author. E-mail: i.m.karabash@gmail.com, karabashi@mail.ru
} 
of-mass motion [22], [25]. The control of spontaneous emission through the Purcell effect is another important application, which requires designing of a cavity with QN eigenvalues close to or far from specially chosen positions [26], [25]. Concerning other applications (including low-threshold lasers, lowpower switches, and adiabatic wavelength conversion), we refer to the vast bibliography in [25], [18].

Since light is essentially difficult to localize, it is hard to realize small-sized optical cavities with strong light confinement. Recently, however, the rapid progress has been made on this issue, see [1], [18] and references therein. As a result, numerical aspects of emerging optimization problems for QN eigenvalues have attracted great current interest among specialists in Applied Mathematics [5], [8], [3].

An attempt to provide an analytical background for QN eigenvalue optimization problems lead the author to the development of the two-parameter perturbation approach [9]. The paper [9] is focused on mathematical details of the proofs, and uses techniques of nonlinear functional analysis and multidimensional complex analysis. The present communication is a continuation of the paper [9]. Its goal is to apply the two-parameter perturbation method to a concrete engineering problem and, using this example, to make the main ideas understandable for specialists in applied sciences. We consider the problem of optimization of a single QN eigenvalue in a one-sided leaky optical cavity. While the corresponding eigenvalue problem is derived under several simplifying assumptions, it is proved to be very useful and have been extensively studied in Physics and numerical papers, see e.g. [24], [16], [5]. We give rigorous mathematical definitions of locally extremal QN eigenvalues and of optimal cavities. In Section 3.2, the existence of such cavities is shown. Then the two-parameter perturbation method is introduced in Section 4 and applied to derive a nonlinear equation for QN modes with extremal properties in Section 6 . We use this nonlinear equation to reduce the cavity's optimization, which is essentially an infinite-dimensional problem, to the four-dimensional problem of finding the zeroes of a specially introduced function $W$, which depends only on one real and one complex variable. The main conclusions are summarized in the last section.

Some of purely mathematical details of the presented method are sketched to make the paper more accessible for non-mathematicians. However, a reader interested in rigorous analytical proofs will be able to recover them without difficulty using the proofs in [9].

Note that, in Engineering and Physics literature, the rate of energy loss is often characterized by other parameters, like Q-factor or lifetime of a QN mode, which in many cases can be approximately expressed in terms of $\operatorname{Im} \omega$ and Re$\omega[15]$. Keeping this in mind, it is easy to rewrite the results of the paper in terms of these, to some extend equivalent, parameters.

\section{Physical settings and the problem statement}

Consider an isotropic non-dispersive transparent medium which has the relative magnetic permeability equal to 1 everywhere, but which is electrically inhomogeneous in one direction. The electromagnetic structure of this medium is described by the (dielectric) permittivity $\varepsilon$, which is $\geq 1$ and varies only in the $x_{3}$-direction $\varepsilon=\varepsilon\left(x_{3}\right)$. When $\varepsilon$ is piecewise constant, this model is called a 1 -D photonic crystal.

Under additional simplifying assumptions that electromagnetic waves pass normally and the electric field $\mathbf{E}$ is in the $x_{2}$-direction $\mathbf{E}=\{0, E, 0\}$, the study of electromagnetic wave propagation can be reduced to the scalar 1-D wave equation

$$
\partial_{x_{3}}^{2} E\left(x_{3}, t\right)=\frac{\varepsilon\left(x_{3}\right)}{c^{2}} \partial_{t}^{2} E\left(x_{3}, t\right),
$$

where the $x_{2}$-component $E$ of the electric field depends only on $x_{3}$, and $c$ is the speed of light in vacuum.

To introduce a one-sided open optical cavity, assume that the medium has the above structure in the slab $0<x_{3}<l$ with piecewise continuous $\varepsilon$ (that is, for a certain partition $0=s_{0}<s_{1}<\cdots<s_{n-1}<$ $s_{n}=l$, the function $\varepsilon(s)$ is continuous in the intervals $s_{j-1}<s<s_{j}$ and has one-sided limits at points $s_{j}$ ). The outer medium is assumed to be a perfect conductor for $x_{3}<0$, and a homogeneous dielectric with constant permittivity $\varepsilon_{\infty} \geq 1$ for $x_{3}>l$. The boundary condition for the electric field on the plane 
$x_{3}=0$ is $E=0$. Since the medium is homogeneous for $x_{3}>l$, the waves radiated from the cavity are never reflected back. If all sources of waves are contained inside the cavity $0<x_{3}<l$, the waves satisfy the radiation boundary condition $\partial_{x_{3}} E+\frac{\sqrt{\varepsilon_{\infty}}}{c} \partial_{t} E=0$ at the interface $x_{3}=l$. For a monochromatic field $E\left(x_{3}, t\right)=E\left(x_{3}\right) e^{-\mathrm{i} \omega t}$ in the cavity, these settings lead to the eigenvalue problem

$$
E^{\prime \prime}\left(x_{3}\right)=-\omega^{2} \frac{\varepsilon\left(x_{3}\right)}{c^{2}} E\left(x_{3}\right)
$$

equipped with the boundary conditions

$$
E(0)=0, \quad \mathrm{i} \omega \frac{\sqrt{\varepsilon_{\infty}}}{c} E(l)=E^{\prime}(l) .
$$

Complex eigen-parameters $\omega=\alpha-\mathrm{i} \beta$ are called quasi-normal (QN) eigenvalues. The real part $\alpha=\operatorname{Re} \omega$ is the frequency of the standing wave $E\left(x_{3}\right) e^{-i \omega t}$. Since the energy leaks from the cavity, the oscillations are decaying. The minus imaginary part $\beta=-\operatorname{Im} \omega$ is positive for all QN eigenvalues and is called the decay rate.

Let $1 \leq \varepsilon_{1}<\varepsilon_{2}$. Restricting ourselves to cavities with $\varepsilon$ in the range

$$
\varepsilon_{1} \leq \varepsilon\left(x_{3}\right) \leq \varepsilon_{2},
$$

consider the following optimization problem: devise, for a given frequency range $\alpha_{1} \leq \alpha \leq \alpha_{2}$, a cavity that has a $Q N$ eigenvalue $\omega=\alpha-\mathrm{i} \beta$ with the minimal possible decay rate $\beta$.

\section{QN eigenvalues: basic properties and extrema's definitions}

\subsection{QN eigenvalues and their multiplicities}

Since the QN eigenvalue problem is one-dimensional, from now on we will write $s$ instead of $x_{3}$. Let the length $l$ of the cavity and the permittivity $\varepsilon_{\infty}$ of the outer medium $s>l$ be fixed. Then the cavity is completely described by the permittivity $\varepsilon(s)$ in the interval $0 \leq s \leq l$ and, for brevity's sake, we will speak on $Q N$ eigenvalues of the cavity $\varepsilon$.

By $\varphi(s)=\varphi(s, z ; \varepsilon)$ and $\psi(s)=\psi(s, z ; \varepsilon)$ we denote the solutions of $E^{\prime \prime}(s)=-z^{2} \frac{\varepsilon(s)}{c^{2}} E(s)$ satisfying

$$
\varphi(0, z ; \varepsilon)=\partial_{s} \psi(0, z ; \varepsilon)=1, \quad \partial_{s} \varphi(0, z ; \varepsilon)=\psi(0, z ; \varepsilon)=0 .
$$

In particular, $\psi$ satisfies the integral equation

$$
\psi(s)=s-\frac{\omega^{2}}{c^{2}} \int_{0}^{s}(s-\tau) \varepsilon(\tau) \psi(\tau) \mathrm{d} \tau, \quad 0 \leq s \leq l .
$$

The QN eigenvalues of $\varepsilon$ are exactly the roots of the equation

$$
F(z)=0, \quad \text { where } F(z)=F(z ; \varepsilon)=\mathrm{i} z \frac{\sqrt{\varepsilon_{\infty}}}{c} \psi(l, z ; \varepsilon)-\partial_{s} \psi(l, z ; \varepsilon) .
$$

The function $F$ is analytic in $z$ in the whole complex plane $\mathbb{C}$. A nontrivial solution $E(s)$ to the problem (2.2), (2.3) is called a $Q N$ mode corresponding to a QN eigenvalue $\omega$. Recall that a solution $E$ is called trivial if $E(s)=0$ for all (more precisely, almost all) $s$ in the interval $0 \leq s \leq l$.

All QN modes of $\varepsilon$ have the form $C \psi(s, \omega ; \varepsilon)$, where $C$ is an arbitrary non-zero constant. Hence the geometric multiplicity of any QN eigenvalue $\omega$ equals 1. In the following, the multiplicity of $\omega$ means its algebraic multiplicity. By definition, the algebraic multiplicity of a QN eigenvalue $\omega$ is its multiplicity as a zero of $F[12]$. The $\omega$-dependent boundary condition $i \omega \frac{\sqrt{\varepsilon_{\infty}}}{c} E(l)=E^{\prime}(l)$ makes the QN eigenvalue problem non-self-adjoint. In particular, QN eigenvalues may be degenerate, i.e., of multiplicity $\geq 2$, see 
e.g. [20], [14], [23], [6]. Concerning the contemporary theory of spectral problems with an eigen-parameter in boundary conditions, we refer to [2] and references therein.

Concerning other properties of QN eigenvalues, it is easy to derive that: (i) the multiplicity of each QN eigenvalue is finite, (ii) the set of QN eigenvalues is symmetric with respect to (w.r.t.) imaginary axis (together with multiplicities), (iii) QN eigenvalues are isolated, $\infty$ is their only possible accumulation point. The property (ii) allows one to restrict the study of QN eigenvalues to the case $\operatorname{Re} \omega \geq 0$.

For a homogeneous cavity with constant permittivity $\varepsilon \geq 1$, the QN eigenvalues are roots of $\tan (-\omega \sqrt{\varepsilon} l / c)=\mathrm{i} \sqrt{\varepsilon / \varepsilon_{\infty}}$. When $\varepsilon \neq \varepsilon_{\infty}$, they are non-degenerate and form a uniformly spaced sequence

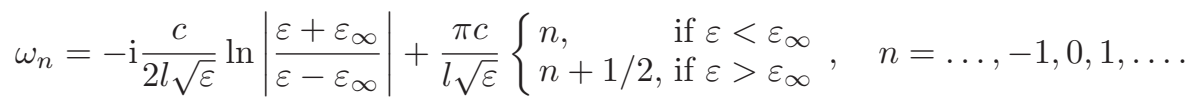

The case when the space outside the cavity is vacuum (i.e., $1=\varepsilon_{\infty} \leq \varepsilon$ ) is discussed in detail in [24]. In the case $\varepsilon=\varepsilon_{\infty}$, there are no QN eigenvalues since the medium is homogeneous for $s>0$ (the energy of the initial disturbance localized in the region $0<s<l$ escapes this region in finite time, see e.g. [4] for detailed explanations).

\subsection{Local and global extrema for admissible frequencies}

The family Adm of admissible cavities is defined by condition (2.4). Mathematically, it is convenient to include in Adm all $L^{\infty}$ functions satisfying (2.4). An admissible direction $h$ for an admissible cavity $\varepsilon$ is a function on the interval $0 \leq s \leq l$ such that the cavity $\varepsilon+h$ is admissible (we call such $h$ a direction since, for all constants $C$ in the range $0<C \leq 1$, the cavity $\varepsilon+C h$ is admissible). We say that $\omega$ is an admissible $Q N$ eigenvalue if it is a $\mathrm{QN}$ eigenvalue of some admissible cavity, and say that $\alpha$ is an admissible frequency if $\alpha=\operatorname{Re} \omega$ for some admissible QN eigenvalue $\omega$.

To get some understanding of admissible frequencies, consider homogeneous cavities. Changing constant $\varepsilon$ in the range $\varepsilon_{1} \leq \varepsilon \leq \varepsilon_{2}$ and taking $\omega_{n}$ given by (3.2), we see that the frequencies $\operatorname{Re} \omega_{n}$ are admissible. They form a sequence of intervals. For $n$ large enough, the interval produced by $\operatorname{Re} \omega_{n}$ overlaps with the next one produced by $\operatorname{Re} \omega_{n+1}$. Hence frequencies $\alpha$ with $|\alpha|$ large enough are admissible. For instance, let us look closer at the case $\varepsilon_{\infty} \leq \varepsilon_{1}$. When constant $\varepsilon$ runs over the interval $\varepsilon_{1}<\varepsilon \leq \varepsilon_{2}$, the real part of $\frac{l \omega_{n}}{\pi c}$ runs over the interval $(n+1 / 2) \varepsilon_{2}^{-1 / 2} \leq \alpha<(n+1 / 2) \varepsilon_{1}^{-1 / 2}$. When $n \geq \frac{1}{\left(\varepsilon_{2} / \varepsilon_{1}\right)^{1 / 2}-1}-\frac{1}{2}$, there is no gap between the $n$-th and $(n+1)$-st intervals. Hence a frequency $\alpha$ is admissible whenever

$$
|\alpha| \geq \frac{\pi c}{l \sqrt{\varepsilon_{2}}}\left(\frac{1}{2}+\left\lceil\frac{1}{\left(\varepsilon_{2} / \varepsilon_{1}\right)^{1 / 2}-1}-\frac{1}{2}\right\rceil\right)
$$

(here $\lceil s\rceil$ is the smallest integer that is greater or equal to $s$ ).

If an admissible cavity $\varepsilon$ has a $Q N$ eigenvalue $\omega$, we say that $\{\omega, \varepsilon\}$ is an admissible pair. The decay rate of an admissible pair is defined by

$$
\operatorname{Dr}(\omega, \varepsilon)=-\operatorname{Im} \omega .
$$

While $\varepsilon$ does not participate in the right hand side, it participates in the domain of definition of the functional Dr. This is essential for the next definition.

We say that an admissible pair $\{\omega, \varepsilon\}$ is a local minimizer of $\operatorname{Dr}$ for a frequency $\alpha$ if, for all sufficiently small admissible directions $\delta \varepsilon$ that keep the frequency of a perturbed QN eigenvalue $\omega+\delta \omega$ of the cavity $\varepsilon+\delta \varepsilon$ equal to $\alpha$, the decay rate of $\{\omega+\delta \omega, \varepsilon+\delta \varepsilon\}$ is $\geq \operatorname{Dr}(\omega, \varepsilon)$. The rigorous form of this condition is: there exist $\delta>0$ such that $\operatorname{Dr}(\widetilde{\omega}, \widetilde{\varepsilon}) \geq \operatorname{Dr}(\omega, \varepsilon)$ for any admissible pair $\{\widetilde{\omega}, \widetilde{\varepsilon}\}$ satisfying the following three conditions

$$
\begin{aligned}
|\widetilde{\varepsilon}(s)-\varepsilon(s)| & <\delta \quad \text { for almost all } 0<s<l, \\
|\widetilde{\omega}-\omega| & <\delta \\
\operatorname{Re} \widetilde{\omega} & =\alpha .
\end{aligned}
$$


Local maximizers of Dr for a particular frequency are defined in a similar way.

We say that $\beta_{\min }(\alpha)$ is a minimal decay rate and $\varepsilon$ is an optimal cavity for a frequency $\alpha$, if the pair $\left\{\alpha-\mathrm{i} \beta_{\min }(\alpha), \varepsilon\right\}$ is admissible and $\beta_{\min }(\alpha) \leq \operatorname{Dr}(\widetilde{\omega}, \widetilde{\varepsilon})$ for any admissible pair $\{\widetilde{\omega}, \widetilde{\varepsilon}\}$ with $\operatorname{Re} \widetilde{\omega}=\alpha$. In other words, such a pair $\left\{\alpha-\mathrm{i} \beta_{\min }(\alpha), \varepsilon\right\}$ is a global minimizer (over the admissible family Adm) of the decay rate for a frequency $\alpha$. We will also say that the corresponding $Q N$ eigenvalue $\alpha-\mathrm{i} \beta_{\min }(\alpha)$ is optimal for the frequency $\alpha$.

Lemma 3.1. For each admissible frequency, there exists an optimal cavity.

The existence of optimal cavities follows from the fact that if admissible QN eigenvalues $\omega_{n}$ approach a complex number $\omega$, then $\omega$ is an admissible QN eigenvalue. To explain this fact, consider admissible cavities $\varepsilon^{(n)}$ that produce the QN eigenvalues $\omega_{n}$. Using the Gronwall-Bellman inequality and assumption (2.4), it is easy to show that the sequence of related $\psi$-solutions $\psi_{n}(s)=\psi\left(s, \omega_{n} ; \varepsilon^{(n)}\right)$ is uniformly bounded in $s$ and $n$ together with the sequences of their $s$-derivatives $\psi_{n}^{\prime}$ and $\psi_{n}^{\prime \prime}$. Hence some subsequence $\psi_{n_{j}}$ converges uniformly to a continuous function $\widetilde{\psi}(s)$ (due to the compact embedding of $W^{2, \infty}[0,1]$ into $C[0,1])$. Then applying the Banach-Alaoglu theorem for $L^{\infty}(0,1)$ to the sequence $\varepsilon^{\left(n_{j}\right)}$, one can pass to a limit in the integral reformulation of (2.2)-(2.3) (see [9], and also [13] for compactness arguments applied to a self-adjoint eigenvalue optimization problem).

\section{Two-parameter perturbations}

We study properties of local extremizers (i.e., local minimizers and maximizers) via the perturbation theory for QN eigenvalues using the following arguments. Assume that $\{\omega, \varepsilon\}$ is a local minimizer. If we consider all small admissible directions $\delta \varepsilon$ that keep $\operatorname{Re}(\omega+\delta \omega)$ equal to $\alpha$, then corresponding corrections $\delta \beta=-\operatorname{Im} \delta \omega$ of the decay rate must be positive. The key point is to show that there are enough various $\delta \varepsilon$ with the above property to impose strong restrictions on extremal $\varepsilon$.

For non-degenerate QN eigenvalues, the first order perturbation correction can be calculated by the standard sensitivity analysis. However our method requires a more delicate approach due to following reasons: (i) QN eigenvalues may be degenerate, (ii) we are interested only in the perturbed QN eigenvalues that stay on the line $\operatorname{Re} \omega=\alpha$. Indeed, even if one is able to find the first order correction that moves a QN eigenvalue along the line $\operatorname{Re} \omega=\alpha$ using the one-parameter perturbation theory for multiple eigenvalues (see e.g. [17] and references therein), there is no any guarantee that higher order corrections do not contribute to the real part of $\omega$.

To resolve these difficulties we study two-parameter perturbations of QN eigenvalues considering them as $z$-roots of the equation $F(z, \varepsilon+\delta \varepsilon)=0$. This requires computation of the (complex) partial derivative $\partial_{z} F(z ; \varepsilon)$ and the directional derivatives $\partial_{\zeta} F(z ; \varepsilon+\zeta h)$ for admissible pairs $\{\omega, \varepsilon\}$. Here $\zeta$ is a complex number, and $h$ is an arbitrary (measurable and essentially bounded) function on $0<s<l$, which is called a direction.

The derivative $\partial_{\zeta} F(z ; \varepsilon+\zeta h)$ exists for an arbitrary direction. More precisely, the Maclaurin series for the solution $\psi$ (see e.g. [7]) can be used to get the Maclaurin series for $F(z ; \varepsilon)$ and, in turn, to show that for arbitrary $n$ directions $h_{1}(s), \ldots, h_{n}(s)$, the function $F\left(\omega+z ; \varepsilon+\zeta_{1} h_{1}+\zeta_{2} h_{2}+\cdots+\zeta_{n} h_{n}\right)$ is an analytic function of $\mathrm{n}+1$ complex variables $z, \zeta_{1}, \ldots, \zeta_{n}$ (moreover, the map $\{z, \varepsilon\} \mapsto F(z ; \varepsilon)$ is analytic on $\left.\mathbb{C} \times L^{\infty}(0,1)\right)$.

Differentiating the integral equation for $\psi$ and solving the produced differential equations, it is possible to express $\partial_{z} F(z ; \varepsilon)$ and $\partial_{\zeta} F(z ; \varepsilon+\zeta h)$ via the fundamental solutions $\varphi$ and $\psi$ (cf. [9]). When $z=\omega$ is 
a $Q N$ eigenvalue of $\varepsilon$, the obtained expressions can be simplified to

$$
\begin{aligned}
\partial_{\zeta} F(\omega ; \varepsilon+\zeta h) & =\frac{\omega^{2}}{c^{2} \psi(l, \omega ; \varepsilon)} \int_{0}^{l} \psi^{2}(s, \omega ; \varepsilon) h(s) \mathrm{d} s, \\
\partial_{z} F(\omega ; \varepsilon) & =\frac{2 \omega}{c^{2} \psi(l, \omega ; \varepsilon)} \int_{0}^{l} \psi^{2}(s, \omega ; \varepsilon) \varepsilon(s) \mathrm{d} s+\mathrm{i} \frac{\sqrt{\varepsilon_{\infty}}}{c} \psi(l, \omega ; \varepsilon) .
\end{aligned}
$$

The last equality yields the following lemma.

Lemma 4.1. A $Q N$ eigenvalue $\omega$ of $\varepsilon$ is degenerate exactly when

$$
\int_{0}^{l} \psi^{2}(s, \omega ; \varepsilon) \varepsilon(s) \mathrm{d} s+\mathrm{i} \frac{c \sqrt{\varepsilon_{\infty}}}{2 \omega} \psi^{2}(l, \omega ; \varepsilon)=0 .
$$

Note that

$$
\psi(l, \omega ; \varepsilon)=\left[i \omega \frac{\sqrt{\varepsilon_{\infty}}}{c} \varphi(l, \omega ; \varepsilon)-\varphi^{\prime}(l, \omega ; \varepsilon)\right]^{-1} \neq 0 .
$$

Let us consider the first-order correction for $\omega$ in the case when $\omega$ is a non-degenerate $Q N$ eigenvalue of $\varepsilon$. Small perturbations of $\varepsilon$ lead to small perturbations of $\omega$ and the perturbed QN eigenvalues remains non-degenerate. Taking $\delta \varepsilon=\zeta h$ with a fixed perturbation direction $h=h(s)$, it is easy to show that the perturbed QN eigenvalue $\omega+\delta \omega$ is an analytic function $\Omega(\zeta)$ of $\zeta$ in a vicinity of $\zeta_{0}=0$. The approximation of $\Omega(\zeta)$ to the first-order term is given by

$$
\Omega(\zeta)=\omega-\zeta \frac{\omega \int_{0}^{l} \psi^{2}(s) h(s) \mathrm{d} s}{2 \int_{0}^{l} \psi^{2}(s) \varepsilon(s) \mathrm{d} s+\mathrm{i} \frac{c \sqrt{\varepsilon_{\infty}}}{\omega} \psi^{2}(l)}+O\left(\zeta^{2}\right) .
$$

This formula, as well as higher order corrections in the non-degenerate case, are known [16].

Consider the case when $\omega$ is degenerate. Perturbations of a multiple eigenvalue may lead to its splitting in several eigenvalues. The general splitting picture is quite complicated and is described by one or several series (Puiseux series) in fractional powers of $\zeta[21],[11],[17]$. For our needs, it is enough to consider only the generic case when the direction $h$ satisfies $\partial_{\zeta} F(\omega ; \varepsilon+\zeta h) \neq 0$. This condition is equivalent to

$$
\int_{0}^{l} \psi^{2}(s, \omega ; \varepsilon) h(s) \mathrm{d} s \neq 0 .
$$

Assume that $\omega$ is a QN eigenvalue of $\varepsilon$ of multiplicity $m$, and that perturbation's direction $h$ satisfies (4.4). To study the equation $F(\omega+z ; \varepsilon+\zeta h)=0$, we introduce the function $Q(z, \zeta)=F(\omega+z ; \varepsilon+\zeta h)$ and write it as a power series in $z$ and $\zeta$. The equation $Q=0$ takes the form

$$
z^{m} \frac{\partial_{z}^{m} Q(0,0)}{m !}+\zeta \partial_{\zeta} Q(0,0)+o\left(z^{m}\right)+o(\zeta)=0
$$

for $z$ and $\zeta$ going to 0 . Note that $\partial_{z}^{m} Q(0,0) \neq 0$ since $\omega$ has multiplicity $m$. Considering $z$ as a function of $\zeta$, we see that $|z(\zeta)| \leq C|\zeta|^{1 / m}$ for $\zeta$ small enough with some constant $C$. So $o\left(z^{m}\right)$ is also $o(\zeta)$. Hence,

$$
z^{m}(\zeta)=-\zeta \frac{m ! \partial_{\zeta} Q(0,0)}{\partial_{z}^{m} Q(0,0)}+o(\zeta)
$$

According to (4.1) and the assumption on $h$,

$$
\partial_{\zeta} Q(0,0)=\frac{\omega^{2}}{c^{2} \psi(l, \omega ; \varepsilon)} \int_{0}^{l} \psi^{2}(s, \omega ; \varepsilon) h(s) \mathrm{d} s \neq 0 .
$$


Taking the $m$-th root, we get a formal first-order correction formula for a perturbed QN eigenvalue $\Omega(\zeta)$

$$
\Omega(\zeta)=\omega+\left(\zeta C_{1}(\omega, \varepsilon, m) \int_{0}^{l} \psi^{2}(s, \omega ; \varepsilon) h(s) \mathrm{d} s\right)^{1 / m}+o\left(|\zeta|^{1 / m}\right),
$$

where

$$
C_{1}(\omega, \varepsilon, m)=-\frac{m ! \omega^{2}}{c^{2} \psi(l, \omega ; \varepsilon) \partial_{z}^{m} F(\omega, \varepsilon)} .
$$

The $m$-th derivative $\partial_{z}^{m} F(\omega, \varepsilon)$ can also be expressed through the fundamental solutions $\varphi$ and $\psi$, but we are not going to use such a refinement in this paper. Note that (4.5) turns into (4.3) for $m=1$.

Now consider two-parameter perturbations of $\omega$, assuming that the directions $h_{1}$ and $h_{2}$ are such that $\partial_{\zeta_{1}} F\left(z ; \varepsilon+\zeta_{1} h_{1}\right)$ and $\partial_{\zeta_{2}} F\left(z ; \varepsilon+\zeta_{2} h_{2}\right)$ are nonzero and

$$
0<\operatorname{Arg} \partial_{\zeta_{2}} F\left(z ; \varepsilon+\zeta_{2} h_{2}\right)-\operatorname{Arg} \partial_{\zeta_{1}} F\left(z ; \varepsilon+\zeta_{1} h_{1}\right)<\pi
$$

for a suitable branch Arg of the arg-function. Here and below $\arg z$ is the complex $\operatorname{argument}$ (phase) of a nonzero complex number $z$. Condition (4.7) means that $\partial_{\zeta_{1}} F\left(z ; \varepsilon+\zeta_{1} h_{1}\right)$ and $\partial_{\zeta_{2}} F\left(z ; \varepsilon+\zeta_{2} h_{2}\right)$, perceived as vectors must not be oppositely directed and, additionally, must be ordered such that (4.7) holds. Considering a perturbed eigenvalue $\omega+z$ of $\varepsilon+\zeta_{1} h_{1}+\zeta_{2} h_{2}$ as a function of $\zeta_{1}$ and $\zeta_{2}$, we write the equation

$$
Q\left(z, \zeta_{1}, \zeta_{2}\right)=0
$$

for the correction $z=z\left(\zeta_{1}, \zeta_{2}\right)$ defining $Q$ as

$$
Q\left(z, \zeta_{1}, \zeta_{2}\right)=F\left(\omega+z, \varepsilon+\zeta_{1} h_{1}+\zeta_{2} h_{2}\right) .
$$

Denote $\zeta=\left\{\zeta_{1}, \zeta_{2}\right\},|\zeta|=\left(\zeta_{1}^{2}+\zeta_{2}^{2}\right)^{1 / 2}$, and $\mathbf{0}=\{0,0,0\}$. When $|\zeta|$ is small, we have

$$
z^{m}=-\zeta_{1} \frac{m ! \partial_{\zeta_{1}} Q(\mathbf{0})}{\partial_{z}^{m} Q \mathbf{0}}-\zeta_{2} \frac{m ! \partial_{\zeta_{2}} Q(\mathbf{0})}{\partial_{z}^{m} Q(\mathbf{0})}+o\left(z^{m}\right)+o\left(\zeta_{1}\right)+o\left(\zeta_{2}\right) .
$$

Assume additionally that

$\zeta_{1}$ and $\zeta_{2}$ are nonnegative and of the same order.

That is, $\zeta_{1}$ and $\zeta_{2}$ go to zero such that their ratios $\zeta_{1} / \zeta_{2}$ and $\zeta_{2} / \zeta_{1}$ are bounded. Under this condition, (4.8) and (4.7) yield that $\left|z\left(\zeta_{1}, \zeta_{2}\right)\right| \leq c|\zeta|^{1 / m}$ for small $|\zeta|$. Hence,

$$
z\left(\zeta_{1}, \zeta_{2}\right)=\left(-\zeta_{1} \frac{m ! \partial_{\zeta_{1}} Q(\mathbf{0})}{\partial_{z}^{m} Q(\mathbf{0})}-\zeta_{2} \frac{m ! \partial_{\zeta_{2}} Q(\mathbf{0})}{\partial_{z}^{m} Q(\mathbf{0})}\right)^{1 / m}+o\left(|\zeta|^{1 / m}\right) .
$$

Appending the values of partial derivatives provided by (4.1), one gets the first-order approximation for perturbed QN eigenvalues

$$
\Omega\left(\zeta_{1}, \zeta_{2}\right)=\omega+\left(C_{1}(\omega, \varepsilon, m) \int_{0}^{l} \psi^{2}(s, \omega ; \varepsilon)\left[\zeta_{1} h_{1}(s)+\zeta_{2} h_{2}(s)\right] \mathrm{d} s\right)^{1 / m}+o\left(|\zeta|^{1 / m}\right)
$$

with $C_{1}$ defined by (4.6).

For sufficiently small positive $\zeta_{1}, \zeta_{2}$ satisfying (4.9), the $m$ branches of $m$-th root produce $m$ different perturbed QN eigenvalues, each of which is non-degenerate. The proof of this fact can be given along the lines suggested in the paper [9], where a slightly different eigenvalue problem was considered. Condition (4.7) ensures that the coefficient under the $m$-th root is nonzero. It is important for the sequel that this coefficient is a linear functional of perturbation directions $h_{1}$ and $h_{2}$.

Formula (4.11) will be applied to the study of the QN eigenvalue optimization via the lemma in the following section. 


\section{Admissible directional derivatives and arguments of eigenvalue corrections}

Let us fix an admissible pair $\{\omega, \varepsilon\}$. The set of admissible directional derivatives of $F$ at $\{\omega, \varepsilon\}$ is defined as the set formed by complex numbers $\partial_{\zeta} F(\omega ; \varepsilon+\zeta h)$ with $h$ running through the set of admissible directions (i.e., we take all $h$ such that $\varepsilon+h$ is an admissible cavity). The set of admissible directional derivatives contains 0 and is convex (that is, if $\xi_{1}$ and $\xi_{2}$ are admissible directional derivatives, then $\gamma \xi_{1}+(1-\gamma) \xi_{2}$ is so for all $\left.0 \leq \gamma \leq 1\right)$. We say that the set of admissible directional derivatives contains a neighborhood of zero if it contains all complex numbers $\xi$ with modulus $|\xi|$ less than a certain positive number. Let $\operatorname{Arg}_{0}$ be the branch of the arg-function that takes values in the interval $-\pi<\operatorname{Arg}_{0} z \leq \pi$.

Lemma 5.1. Assume that the set of admissible directional derivatives of $F$ at an admissible pair $\{\omega, \varepsilon\}$ contains a neighborhood of zero. Then for any $\theta$ in the range $-\pi<\theta \leq \pi$, there exists a sequence of admissible pairs $\left\{\omega_{n}, \varepsilon^{(n)}\right\}$ such that

(i) $\operatorname{Arg}_{0}\left(\omega_{n}-\omega\right)=\theta$ for all $n=1,2, \ldots$,

(ii) $\omega_{n}$ tends to $\omega$ and $\varepsilon^{(n)}$ tends to $\varepsilon$ (the latter means that the essential suprema of $\left|\varepsilon(s)-\varepsilon^{(n)}(s)\right|$ tends to zero as $n$ goes to $\infty)$.

In particular, the lemma yields that if $\{\omega, \varepsilon\}$ is a local extremizer of Dr for the frequency $\operatorname{Re} \omega$, then the set of admissible directional derivatives of $F$ at $\{\omega, \varepsilon\}$ cannot contain a neighborhood of zero.

To derive the lemma, note that its assumptions allows us to chose admissible directions $h_{1}$ and $h_{2}$ and the branch of the m-th root in (4.11) such that

(A1) assumption (4.7) is valid for $h_{1}$ and $h_{2}$,

(A2) for positive $\zeta$, the corresponding first order corrections to $\omega$,

$$
\left(\zeta C_{1}(\omega, \varepsilon, m) \int_{0}^{l} \psi^{2}(s, \omega ; \varepsilon) h_{1}(s) \mathrm{d} s\right)^{1 / m} \text { and }\left(\zeta C_{1}(\omega, \varepsilon, m) \int_{0}^{l} \psi^{2}(s, \omega ; \varepsilon) h_{2}(s) \mathrm{d} s\right)^{1 / m}
$$

have the arguments slightly smaller and, respectively, slightly greater than $\theta$,

(A3) the chosen branch of the m-th root is continuous in the (infinite) cone of complex plane covered by numbers

$$
C_{1}(\omega, \varepsilon, m) \int_{0}^{l} \psi^{2}(s, \omega ; \varepsilon)\left[\zeta_{1} h_{1}(s)+\zeta_{2} h_{2}(s)\right] \mathrm{d} s
$$

while $\zeta_{1}$ and $\zeta_{2}$ run through positive numbers.

This allows us to apply formula (4.11), which yields that the argument $\operatorname{Arg}\left(\Omega\left(\zeta_{1}, \zeta_{2}\right)-\omega\right)$ of the QN eigenvalue correction changes continuously for small positive $\zeta_{1}$ and $\zeta_{2}$. This and (A2) imply that for small enough $\epsilon$ there exist positive $\zeta_{1}$ and $\zeta_{2}$ such that $\zeta_{1}+\zeta_{2}=\epsilon$ and $\operatorname{Arg}\left(\Omega\left(\zeta_{1}, \zeta_{2}\right)-\omega\right.$ ) equals $\theta$ (up to a multiple of $2 \pi$ ). Finally, we take such $\zeta_{1}, \zeta_{2}$ for a sequence $\epsilon_{n}$ going to 0 . Note that the convexity of the families of admissible cavities and admissible directions is essentially used here.

\section{Nonlinear eigenvalue problem for local extrema}

In this section we derive a nonlinear eigenvalue problem for QN modes corresponding to local extremizers.

Lemma 6.1. Let $\varepsilon$ be an admissible cavity and $\operatorname{Im} z^{2}<0$. Then $\psi(s, z ; \varepsilon) \neq 0$ in the interval $0<s \leq l$, and the function $\xi(s)=\arg \psi^{2}(s, z ; \varepsilon)$ is strictly increasing (the values of arg are chosen such that $\xi$ is continuous for $0<s \leq l$ ). 
Let us show this. Assume first that $\psi\left(s_{1}\right)=0$, where $\psi(s)=\psi(s, z ; \varepsilon)$. Then

$$
z^{2} \int_{0}^{s_{1}}|\psi(s)|^{2} \varepsilon(s) \mathrm{d} s=-c^{2} \int_{0}^{s_{1}} \psi^{\prime \prime}(s) \overline{\psi(s)} \mathrm{d} s=-c^{2} \int_{0}^{s_{1}}\left|\psi^{\prime}(s)\right|^{2} \mathrm{~d} s .
$$

The number in the right-hand side is real, but by our assumptions $z^{2}$ is not real. Hence $s_{1}=0$ (note that $\psi^{\prime}(0)=1$ and so $\psi$ is non-zero for small positive $\left.s\right)$. Thus, $\psi(s) \neq 0$ for $s>0$. Note that

$$
\xi^{\prime}(s)=2 \operatorname{Im}(\ln \psi(s))^{\prime}=2 \operatorname{Im} \frac{\psi^{\prime}(s) \overline{\psi(s)}}{|\psi(s)|^{2}} .
$$

This shows that, on the one hand, the function $|\psi|^{2} \xi^{\prime}$ tends to 0 as $s$ goes to $0^{+}$, and on the other hand,

$$
\left(|\psi(s)|^{2} \xi^{\prime}(s)\right)^{\prime}=2 \operatorname{Im} \psi^{\prime \prime}(s) \overline{\psi(s)}=-2 c^{-2} \varepsilon(s)|\psi(s)|^{2} \operatorname{Im} z^{2}>0 .
$$

Hence $|\psi|^{2} \xi^{\prime}$ is positive for $0<s \leq l$, and so is $\xi^{\prime}$.

Denote by $\chi_{a, b}$ the function that equals 1 in the interval $a<s<b$ and equals 0 outside this interval. For a finite number of complex numbers $z_{1}, z_{2}, \ldots, z_{n}$, the convex hull of these numbers, by definition, consists of numbers $c_{1} z_{1}+c_{2} z_{2}+\ldots c_{n} z_{n}$, where $c_{1}+c_{2}+\cdots+c_{n}=1$ and all $c_{j}$ are nonnegative. Clearly, a set of complex numbers is convex if and only if it contains a convex hull of every its finite subset.

Assume that $\varepsilon$ is a piecewise continuous admissible cavity, and that $\omega$ with $\operatorname{Re} \omega>0$ is a QN eigenvalue of $\varepsilon$. Since changing of $\varepsilon$ at a finite number of points does not influence QN eigenvalues, this is not an essential restriction to assume from now on that $\varepsilon$ takes either its limit from the left or its limit from the right at the points of discontinuity and the endpoints $s=0$ and $s=l$.

Further, suppose that $\varepsilon_{1}<\varepsilon(s)<\varepsilon_{2}$ in a certain interval $s_{1}<s<s_{2}$. Then

$$
\varepsilon_{1}+\delta_{1}<\varepsilon(s)<\varepsilon_{2}-\delta_{1} \text { in some narrower interval } s_{3}<s<s_{4}
$$

for small enough $\delta_{1}>0$. Hence, the two directions $\delta_{1} \chi_{a, b}$ and $(-1) \delta_{1} \chi_{a, b}$ are admissible for every $a$ and $b$ such that $s_{3}<a<b<s_{4}$. It follows from Lemma 6.1 that there exist $a_{1}, a_{2}, b_{1}, b_{2}$ with the following two properties:

(i) $s_{3}<a_{1}<b_{1}<a_{2}<b_{2}<s_{4}$,

(ii) the directional derivatives $\partial_{\zeta} F\left(z ; \varepsilon+\zeta \chi_{a_{1}, b_{1}}\right), \partial_{\zeta} F\left(z ; \varepsilon+\zeta \chi_{a_{2}, b_{2}}\right)$ are non-zero and the difference of their arguments is not a multiple of $\pi$.

With such a choice of $a_{1,2}$ and $b_{1,2}$, the convex hull of the four admissible directional derivatives $\partial_{\zeta} F(z ; \varepsilon \pm$ $\left.\zeta \delta_{1} \chi_{a_{1}, b_{1}}\right), \partial_{\zeta} F\left(z ; \varepsilon \pm \zeta \delta_{1} \chi_{a_{2}, b_{2}}\right)$ contains a neighborhood of zero. Since the set of admissible directional derivatives is convex, it also contains a neighborhood of zero. By Lemma 5.1 the pair $\{\omega, \varepsilon\}$ is not a local extremizer.

Thus, we have shown that if an admissible pair $\{\omega, \varepsilon\}$ (with piecewise continuous $\varepsilon$ ) is a local extremizer, then $\varepsilon$ takes only the extreme possible values $\varepsilon_{1}$ and $\varepsilon_{2}$.

Since the set of admissible directional derivatives at $\{\omega, \varepsilon\}$ is convex, it either contains a neighborhood of zero or, otherwise,

the set of nonzero admissible directional derivatives is contained in

$$
\text { a certain complex half-plane } \theta_{0} \leq \arg z \leq \theta_{0}+\pi \text {. }
$$

Assume again that $\{\omega, \varepsilon\}$ is a local extremizer and that $\varepsilon$ is piecewise continuous. Then Lemma 5.1 yields that the case (6.2) takes place. We have shown before that $\varepsilon$ takes only values $\varepsilon_{1}$ and $\varepsilon_{2}$. Now, repeating the use of Lemma 5.1, we want to find restrictions on the points $s$ where such an extreme $\varepsilon$ switches between $\varepsilon_{1}$ and $\varepsilon_{2}$. To this purpose, consider directional derivatives produced by directions $\pm \delta_{1} \chi_{a, b}$ with intervals $a<s<b$ of small lengths. For any point $s_{0}$, there exists an interval (of positive 
length) $s_{1} \leq s \leq s_{2}$ containing $s_{0}$ and such that $\varepsilon$ is constant on it. To be specific, assume $\varepsilon\left(s_{0}\right)=\varepsilon_{1}$. Then for any subinterval $a \leq s \leq b$ of the interval $s_{1} \leq s \leq s_{2}$, the direction $\delta_{1} \chi_{a, b}$ is admissible whenever $0 \leq \delta_{1} \leq \varepsilon_{2}-\varepsilon_{1}$. The corresponding admissible directional derivative $\partial_{\zeta} F\left(\omega, \varepsilon+\zeta \delta_{1} \chi_{a, b}\right)$ equals

$$
\delta_{1} \frac{\omega^{2}}{c^{2} \psi(l, \omega ; \varepsilon)} \int_{a}^{b} \psi^{2}(s, \omega ; \varepsilon) \mathrm{d} s .
$$

Taking $a$ and $b$ close enough to $s_{0}$, it is possible to make the difference of arguments

$$
\arg \partial_{\zeta} F\left(\omega, \varepsilon+\zeta \delta_{1} \chi_{a, b}\right)-\arg \left(\frac{\omega^{2}}{c^{2} \psi(l, \omega ; \varepsilon)} \psi^{2}\left(s_{0}, \omega ; \varepsilon\right)\right)
$$

arbitrary small (after possible correction on a multiple of $2 \pi$ ). Combining this with (6.2), one can see that

$$
\theta_{0} \leq \arg \left(\frac{\omega^{2}}{c^{2} \psi(l, \omega ; \varepsilon)} \psi^{2}\left(s_{0}, \omega ; \varepsilon\right)\right) \leq \theta_{0}+\pi
$$

Similarly, if $\varepsilon\left(s_{0}\right)=\varepsilon_{2}$, then there exist $a$ and $b$ arbitrary close to $s_{0}$ such that the direction $(-1) \delta_{1} \chi_{a, b}$ is admissible whenever $0 \leq \delta_{1} \leq \varepsilon_{2}-\varepsilon_{1}$. This yields

$$
\theta_{0}-\pi \leq \arg \left(\frac{\omega^{2}}{c^{2} \psi(l, \omega ; \varepsilon)} \psi^{2}\left(s_{0}, \omega ; \varepsilon\right)\right) \leq \theta_{0} .
$$

Summarizing, we see that, for a local extremizer $\{\omega, \varepsilon\}$ with piecewise continuous $\varepsilon$, there exists $\theta_{1}$ such that $\varepsilon$ takes the value $\varepsilon_{1}$ in the intervals where $\theta_{1}<\arg \psi^{2}(s, \omega ; \varepsilon)<\theta_{1}+\pi$, and takes the value $\varepsilon_{2}$ in the intervals where $\theta_{1}-\pi<\arg \psi^{2}(s, \omega ; \varepsilon)<\theta_{1}$ (here, the double inequalities with multi-valued arg function are assumed to be valid if they are valid for one of its values). The values of $\varepsilon$ at the finite set of points where $\psi^{2}(s)$ cross the lines $\arg z=\theta_{1}$ and $\arg z=\theta_{1}+\pi$ are not important since these values do not influence QN eigenvalues of $\varepsilon$. The angle $\theta_{1}$ is related to $\theta_{0}$ of (6.2) through

$$
\theta_{1}=\theta_{0}-\arg \frac{\omega^{2}}{c^{2} \psi(l, \omega ; \varepsilon)} .
$$

Similar arguments also works for the case $\operatorname{Re} \omega<0$ (for instance, trough the use of the symmetry of $\mathrm{QN}$ eigenvalues w.r.t. $\mathrm{i} \mathbb{R}$ ) and for the case $\operatorname{Re} \omega=0$ (for this case some modifications are needed, but considerations are simpler since the solution $\psi$ is real-valued). Finally, note that the assumption of piecewise continuity of $\varepsilon$ can be dropped (that is, $\varepsilon$ can be assumed only to be an admissible $L^{\infty}$-function). This can be done if instead of small intervals $a<s<b$, one considers sets of small measure and small diameter. Corresponding modifications require a number of purely technical details, which a concerned reader can recover without difficulties using [9].

It is convenient to write the obtained result in terms of a nonlinear eigenvalue problem. Denote by $\{\operatorname{Im} \zeta>0\}$ the (open) upper complex half-plane, which, by definition, consists of complex numbers $\zeta$ with positive imaginary part. Similarly the half-plane $\{\operatorname{Im} \zeta<0\}$ consists of numbers $\zeta$ such that $\operatorname{Im} \zeta<0$. Let $\chi_{\operatorname{Im} \zeta>0}(z)=1$ when $\operatorname{Im} z>0$, and $\chi_{\operatorname{Im} \zeta>0}(z)=0$ when $\operatorname{Im} z \leq 0$. So $\chi_{\operatorname{Im} \zeta>0}$ is the indicator function (characteristic function) of $\{\operatorname{Im} \zeta>0\}$.

Theorem 6.2. Assume that $\{\omega, \varepsilon\}$ is a local extremizer (i.e., minimizer or maximizer) of the decay rate for an admissible frequency $\alpha=\operatorname{Re} \omega$. Then the boundary value problem (2.3) for the nonlinear equation

$$
E^{\prime \prime}=-\frac{\omega^{2}}{c^{2}} E\left[\varepsilon_{1}+\left(\varepsilon_{2}-\varepsilon_{1}\right) \chi_{\operatorname{Im} \zeta>0}\left(E^{2}\right)\right]
$$

has a nontrivial solution $E(s)$ satisfying

$$
\varepsilon(s)=\varepsilon_{1}+\left(\varepsilon_{2}-\varepsilon_{1}\right) \chi_{\operatorname{Im} \zeta>0}\left(E^{2}(s)\right) \quad \text { (almost everywhere) on } 0<s<l .
$$


Let us show this for the case $\operatorname{Re} \omega>0$. Multiplying the solution $\psi$ to a constant $e^{\mathrm{i}\left(\pi-\theta_{1}\right) / 2}$ with $\theta_{1}$ from (6.3), one gets another solution $E(s)=e^{\mathrm{i}\left(\pi-\theta_{1}\right) / 2} \psi(s, \omega ; \varepsilon)$ to the linear equation $E^{\prime \prime}=-\frac{\omega^{2}}{c^{2}} \varepsilon E$. This solution additionally satisfies (2.3) and has the property that $E^{2}$ takes values in $\{\operatorname{Im} \zeta<0\}$ when $\varepsilon(s)$ equals $\varepsilon_{1}$, and takes values in $\{\operatorname{Im} \zeta>0\}$ when $\varepsilon(s)=\varepsilon_{2}$ (after a possible correction of $\varepsilon$ at a finite number of points). So $\varepsilon$ and $E$ are additionally connected by (6.5) and, therefore, $E$ is a solution to (6.4). Modifications of this proof for the cases $\operatorname{Re} \omega=0$ and $\operatorname{Re} \omega<0$ are similar to that of [9].

Note that if $y(s)$ is a solution to the equation

$$
E^{\prime \prime}=-\frac{z^{2}}{c^{2}} E\left[\varepsilon_{1}+\left(\varepsilon_{2}-\varepsilon_{1}\right) \chi_{\operatorname{Im} \zeta>0}\left(E^{2}\right)\right],
$$

then $C y(s)$ is so for each positive or negative constant $C$. This implies that any nontrivial solution $y$ to (6.6) satisfying $y(0)=0$ can be written in the form $y(s)=C \Psi(s ; z, \theta)$ with a real nonzero constant $C$ and a function $\Psi(s ; z, \theta)$ defined by

$$
\partial_{s}^{2} \Psi=-\frac{z^{2}}{c^{2}} \Psi\left[\varepsilon_{1}+\left(\varepsilon_{2}-\varepsilon_{1}\right) \chi_{\operatorname{Im} \zeta>0}\left(\Psi^{2}\right)\right], \quad \Psi(0 ; z, \theta)=0, \quad \partial_{s} \Psi(0 ; z, \theta)=e^{\mathrm{i} \theta},
$$

where $z$ is a complex number and $\theta$ is a number in the interval $0<\theta \leq \pi$. It is easy to see that, for the initial data $E(0), E^{\prime}(0)$ such that $E(0)=0$ and $E^{\prime}(0) \neq 0$, equation (6.6) has a unique solution, and so $\Psi$ is well-defined.

For $0<\theta \leq \pi$ and a complex number $z$, define the function

$$
W(z, \theta)=\mathrm{i} z \frac{\sqrt{\varepsilon_{\infty}}}{c} \Psi(l ; z, \theta)-\partial_{s} \Psi(l ; z, \theta) .
$$

One can see that the nonlinear eigenvalue problem (6.4), (2.3) has a nontrivial solution exactly for those numbers $\omega$ that satisfy

$$
W(\omega, \theta)=0 \text { for at least one } \theta .
$$

Let (6.7) be valid with a certain $\theta$. Then $y(s)=\Psi(s ; \omega, \theta)$ is a solution to the original linear eigenvalue problem (2.2), (2.3) with admissible $\varepsilon(s)=\varepsilon_{1}+\left(\varepsilon_{2}-\varepsilon_{1}\right) \chi_{\operatorname{Im} \zeta>0}\left(y^{2}\right)$, and $\omega$ is a QN eigenvalue of $\varepsilon$. Thus, $\omega$ is an admissible QN eigenvalue. So Theorem 6.2 and the definitions of optimal cavities and QN eigenvalues (see Sect.3.2) yield our main result.

Theorem 6.3. The minimal decay rate $\beta_{\min }(\alpha)$ for an admissible frequency $\alpha$ equals to the minimal real number $\beta$ having the property that $W(\alpha-\mathrm{i} \beta, \theta)=0$ for at least one $\theta$ in the range $0<\theta \leq \pi$.

Moreover, let $\omega=\alpha-\mathrm{i} \beta_{\min }(\alpha)$ be the corresponding optimal $Q N$ eigenvalue. Then for any $\theta$ such that $W(\omega, \theta)=0$, the cavity defined by

$$
\varepsilon(s)=\varepsilon_{1}+\left(\varepsilon_{2}-\varepsilon_{1}\right) \chi_{\operatorname{Im} \zeta>0}\left(\Psi^{2}(s ; \omega, \theta)\right)
$$

is optimal for the frequency $\alpha$. And vice versa, for each cavity $\varepsilon$ optimal for $\alpha$, there exists $\theta$ such that (6.8) holds.

\section{Conclusions and the discussion of related spectral problems}

Conclusions. The paper is concerned with QN eigenvalues of 1-D leaking optical cavities. The cavities are described by dielectric permittivity function $\varepsilon$ which depends on one variable $x_{3}$ and is assumed to take values in a fixed range $\varepsilon_{1} \leq \varepsilon\left(x_{3}\right) \leq \varepsilon_{2}$ for $0<x_{3}<l$. The length $l$ of the cavity is fixed. Cavities satisfying these restrictions are called admissible. 
We analytically study cavities that produce QN eigenvalues $\omega$ with a given frequency $\operatorname{Re} \omega=\alpha$ and locally extremal (locally maximal or minimal for this frequency) decay rate $\beta=-\operatorname{Im} \omega$. We show that cavities with such locally extremal properties are 1-D photonic crystals consisting of alternating layers of two materials with two extreme allowed dielectric permittivities $\varepsilon_{1}$ and $\varepsilon_{2}$. This explains effects observed in numerical experiments for very kindred (but slightly different) optimization problems [8], [5]. To find thicknesses of the layers in extremal cavities, we derive a nonlinear eigenvalue problem for their QN modes. It occurs that $x_{3}$ coordinates of the interface planes between the layers are tied to rotation in the complex plane (i.e., to the arg-function) of the corresponding extremal QN mode.

For each admissible frequency $\alpha$ there exists a cavity that creates a QN eigenvalue with minimal possible decay rate. In the paper, this QN eigenvalue is called optimal for the frequency $\alpha$. We show that such optimal QN eigenvalues can be easily found via zeroes of a function $W$, which is defined by $W(z, \theta)=\mathrm{i} z \frac{\sqrt{\varepsilon_{\infty}}}{c} \Psi(l)-\Psi^{\prime}(l)$. Here $\Psi(s)=\Psi(s ; z, \theta)$ is a solution to the equation

$$
y^{\prime \prime}=-\frac{z^{2}}{c^{2}} y\left[\varepsilon_{1}+\left(\varepsilon_{2}-\varepsilon_{1}\right) \chi_{\operatorname{Im} \zeta>0}\left(y^{2}\right)\right]
$$

satisfying the initial conditions

$$
y(0)=0, \quad y^{\prime}(0)=e^{\mathrm{i} \theta},
$$

and $\chi_{\operatorname{Im} \zeta>0}$ is the indicator function (characteristic function) of the upper complex half-plane $\operatorname{Im} \zeta>0$. So the function $W$ depends only on two variables (the complex variable $z$ and the real variable $\theta, 0<\theta \leq \pi$ ) and can be easily calculated by numerical methods. This effectively excludes the unknown optimal dielectric permittivity function $\varepsilon$ from the process of calculation of an optimal QN eigenvalue. After finding an optimal QN eigenvalue $\omega$ from the equation $W(\omega, \theta)=0$, the corresponding QN modes $\Psi(s ; \omega, \theta)$ can be obtained by solving (7.1)-(7.2). Optimal dielectric permittivity functions can be recovered using the formula $\varepsilon(s)=\varepsilon_{1}+\left(\varepsilon_{2}-\varepsilon_{1}\right) \chi_{\operatorname{Im} \zeta>0}\left(\Psi^{2}(s ; \omega, \theta)\right)$.

Discussion. Note that it is not clear whether it is possible that there are non-equivalent optimal $\varepsilon(s)$ corresponding to certain $\alpha$. This can happen if $W(\omega, \theta)=0$ for several different $\theta$. For a related, but different, optimization problem, the uniqueness question was studied in [10] (see also [9, Remark 2.1]).

For applications discussed in [22], [26], a specific frequency range, where a QN eigenvalue with a very low decay rate has to be created, is important. The frequency restriction $\alpha_{1} \leq \alpha \leq \alpha_{2}$ is also mathematically convenient because it essentially simplifies the question of existence of optimal QN eigenvalue, see the proof of Lemma 3.1 above.

Dropping the frequency restriction in the above problem, one comes to the question of maximization of the spectral abscissa $\mu(\varepsilon):=\sup _{n} \operatorname{Im} \omega_{n}$ (where $\left\{\omega_{n}\right\}$ is a set of all QN eigenvalues of the cavity $\varepsilon)$. Presently the author does not know any applications of the problem of maximization of spectral abscissa. Of course, this does not mean that such applications do not exist. The quantity $2|\mu(\varepsilon)|$ can be interpreted as the rate of decay of the energy in the cavity for a generic initial disturbance of the electromagnetic field, cf. [4, Sect.1]. The question of minimization of $\mu(\varepsilon)$ is important in the studies of dumped mechanical systems. The existence of corresponding optimal design was considered in [4] for a class of twice differentiable $\varepsilon$. However, the problem of maximization of $\mu(\varepsilon)$ for $L^{\infty}$-functions $\varepsilon(s)$ satisfying (2.4) is quite different and leads to a circle of Spectral Theory questions that are not well studied. For instance, it is not clear whether there exists an admissible $\varepsilon$ with $\mu(\varepsilon)=0$. The upper bound on $\mu(\varepsilon)$ obtained in [4] suggests that such $\varepsilon$ must have infinite total variation. It seems that for $\varepsilon(s)$ that are not twice differentiable, possible asymptotics of $\omega_{n}$ were not investigated (see [6] and [20] concerning this question for $\varepsilon$ in the Sobolev space $\left.W^{2,2}[0, l]\right)$.

On the other side, if one interprets QN eigenvalues as resonances of a nonhomogeneous dumped string with the density $B(s)=\frac{\varepsilon(s)}{c^{2}}$ (see e.g. [14], [4], [20]), it makes sense to consider strings that bear atom masses at certain points. Recently Vyacheslav Pivovarchik has informed the author that the results of [19] on asymptotics of $\omega_{n}$ yield that the spectral abscissa equals zero for each string with uniformly positive density $B \in W^{4,2}[0, l]$ and an additional atom mass at the right end $s=l$. 
Acknowledgements. The author is grateful to Igor Chueshov, Robert Kohn, Vyacheslav Pivovarchik, and Roald Trigub for interest to this research and stimulating discussions, and to Andrey Shishkov for constant moral support.

\section{References}

[1] Y. Akahane, T. Asano, B. Song, S. Noda. High-Q photonic nanocavity in a two-dimensional photonic crystal. Nature, 425 (2003), 944-947.

[2] S. Albeverio, R. Hryniv, Ya. Mykytyuk. Inverse spectral problems for coupled oscillating systems: reconstruction from three spectra. Methods Funct. Anal. Topology, 13 (2007), No. 1, 110-123.

[3] S. Burger, J. Pomplun, F. Schmidt, L. Zschiedrich. Finite-element method simulations of high-Q nanocavities with $1 D$ photonic bandgap. Proc. SPIE Vol. 7933 (2011), 79330T (Physics and Simulation of Optoelectronic Devices XIX).

[4] S. Cox, E. Zuazua. The rate at which energy decays in a string damped at one end. Indiana Univ. Math. J., 44 (1995), No. 2, 545-573.

[5] P. Heider, D. Berebichez, R.V. Kohn, M.I. Weinstein. Optimization of scattering resonances. Struct. Multidisc. Optim., 36 (2008), 443-456.

[6] G.M. Gubreev, V.N. Pivovarchik. Spectral analysis of the Regge problem with parameters. Funktsional. Anal. i Prilozhen., 31 (1997), No. 1, 70-74 (Russian); Engl. transl.: Funct. Anal. Appl., 31 (1997), No. 1, 54-57.

[7] I.S. Kac, M.G. Krein. On the spectral functions of the string. Supplement II in Atkinson, F. Discrete and continuous boundary problems. Mir, Moscow, 1968. Engl. transl.: Amer. Math. Soc. Transl., Ser. 2, 103 (1974), $19-102$.

[8] C.-Y. Kao, F. Santosa. Maximization of the quality factor of an optical resonator. Wave Motion, 45 (2008), $412-427$.

[9] I.M. Karabash. Optimization of quasi-normal eigenvalues for 1-D wave equations in inhomogeneous media; description of optimal structures. To appear in Asymptotic Analysis (see also the preprint of the paper arXiv:1103.4117v5 [math.SP]).

[10] I.M. Karabash. Optimization of quasi-normal eigenvalues for Krein-Nudelman strings. Integral Equations and Operator Theory, DOI: 10.1007/s00020-012-2014-4

[11] T. Kato. Perturbation theory for linear operators. Springer-Verlag, Berlin-Heidelberg-New York, 1980.

[12] M.V. Keldysh. On the characteristic values and characteristic functions of certain classes of non-self-adjoint equations. Doklady Akad. Nauk SSSR, 77 (1951), 11-14 (Russian).

[13] M.G. Krein. On certain problems on the maximum and minimum of characteristic values and on the Lyapunov zones of stability. Prikl. Mat. Meh., 15 (1951), 323-348 (Russian); English transl.: Amer. Math. Soc. Transl.(2), 1 (1955), $163-187$.

[14] M.G. Krein, A.A. Nudelman. On direct and inverse problems for the boundary dissipation frequencies of a nonuniform string. Dokl. Akad. Nauk SSSR, 247 (1979), No. 5, 1046-1049 (Russian). Engl. transl.: Soviet Math. Dokl., 20 (1979), No. 4, 838-841.

[15] L.D. Landau, E.M. Lifshitz. Electrodynamics of continuous media. Pergamon, 1984.

[16] P.T. Leung, S.Y. Liu, S.S. Tong, K. Young. Time-independent perturbation theory for quasinormal modes in leaky optical cavities. Phys. Rev. A, 49 (1994), 3068-3073.

[17] J. Moro, J.V. Burke, M.L. Overton. On the Lidskii-Vishik-Lyusternik perturbation theory for eigenvalues of matrices with arbitrary Jordan structure. SIAM J. Matrix Anal. Appl., 18 (1997), No. 4, 793-817.

[18] M. Notomi, E. Kuramochi, H. Taniyama. Ultrahigh-Q nanocavity with 1d photonic gap. Opt. Express, 16 (2008), 11095.

[19] V.N. Pivovarchik, Inverse problem for a smooth string with damping at one end. J. Operator Theory, 38 (1997), No. 2, 243-263.

[20] V. Pivovarchik, C. van der Mee. The inverse generalized Regge problem. Inverse Problems, 17 (2001), No. 6, $1831-1845$.

[21] M. Reed, B. Simon. Methods of modern mathematical physics. IV. Analysis of operators. Academic Press, New YorkLondon, 1978.

[22] G. Rempe. One atom in an optical cavity: spatial resolution beyond the standard diffraction limit. Appl. Phys. B, 60 (1995), 233-237.

[23] M.A. Shubov. Spectral operators generated by damped hyperbolic equations. Integral Equations Operator Theory, 28 (1997), No. 3, 358-372.

[24] K. Ujihara. Quantum theory of a one-dimensional optical cavity with output coupling. Field quantization. Phys. Rev. A, 12 (1975), 148-158.

[25] K.J. Vahala. Optical microcavities. Nature, 424 (2003), 839-846.

[26] Y. Yamamoto, F. Tassone, H. Cao. Semiconductor cavity quantum electrodynamics. Springer, New York, 2000. 